\title{
Carboinsuflação em úlceras crônicas dos membros inferiores
}

\section{Carbon insufflations in chronic ulcers of the lower limbs}

Antonio Carlos Abramo ${ }^{1}$

Tatiana Tais Teixeira ${ }^{2}$

Trabalho realizado no ACA Instituto de Assistência em Cirurgia Plástica de São Paulo, São Paulo, SP, Brasil.

Artigo submetido no SGP (Sistema de Gestão de Publicações) da RBCP.

Artigo recebido: 20/2/2011 Artigo aceito: $27 / 5 / 2011$

\begin{abstract}
RESUMO
Introdução: A infusão controlada do $\mathrm{CO}_{2}$ recupera o tecido de granulação em úlceras crônicas por insuficiência venosa dos membros inferiores, facilitando a integração do enxerto autólogo de pele. Método: Foram avaliadas ulcerações com evolução média de 12,5 meses. A infusão controlada do $\mathrm{CO}_{2}$ foi aplicada a $0,5 \mathrm{~cm}$ da borda da ferida, com a agulha voltada para o tecido de granulação. As puncturas foram realizadas a cada $2,5 \mathrm{~cm}$. As sessões foram repetidas com intervalos de 72 horas. Biopsias foram realizadas no tecido de granulação antes e após a infusão do $\mathrm{CO}_{2}$. Resultados: Observou-se aumento numérico de capilares, macrófagos e fibroblastos no tecido de granulação após a infusão controlada do $\mathrm{CO}_{2} \cdot \mathrm{O}$ aumento médio do diâmetro das arteríolas pré-capilares foi de 3,4 vezes. Conclusão: A infusão controlada do $\mathrm{CO}_{2}$ estimulou a fibrogênese e promoveu uma resposta vasomotora e celular na ferida, facilitando o fechamento espontâneo das úlceras de membros inferiores ou tornando-as aptas a receber o enxerto autólogo de pele.
\end{abstract}

Descritores: Dióxido de Carbono. Vasodilatação. Úlcera da Perna. Úlcera.

\begin{abstract}
Introduction: $\mathrm{CO}_{2}$ infusion-controlled restores granulation tissue in chronic venous caused by venous insufficiency of the lower limbs, becoming ready to receive autologous skin grafting. Methods: Mean time of ulceration was 12.5 months. $\mathrm{CO}_{2}$ infusion-controlled was applied $0.5 \mathrm{~cm}$ from the wound edge. Punctures were done $2.5 \mathrm{~cm}$ distant from each other along the border of the wound with the needle pointed toward the granulation tissue. Biopsies were taken from the granulation tissue before and after $\mathrm{CO}_{2}$ application. The sessions were performed at intervals of 72 hours. Results: Noticeable increase of capillaries, macrophages and fibroblasts were founded into the granulation tissue after $\mathrm{CO}_{2}$ infusioncontrolled. Diameter of pre-capillary arterioles increased 3.4 times after $\mathrm{CO}_{2}$ infusioncontrolled. Conclusion: $\mathrm{CO}_{2}$ infusion-controlled restores the blood flow in chronic wound of the lower limbs, promoting its spontaneous closure or improving granulation tissue ready to receive autologous skin grafting.
\end{abstract}

Keywords: Carbon Dioxide. Vasodilatation. Leg Ulcer. Ulcer.

1. Membro Titular da Sociedade Brasileira de Cirurgia Plástica; Regente do Serviço ACA - Instituto de Assistência em Cirurgia Plástica de São Paulo; Mestrado e Doutorado em Cirurgia Plástica pela Escola Paulista de Medicina, Universidade Federal de São Paulo - UNIFESP, São Paulo, SP, Brasil.

2. Estagiária do ACA - Instituto de Assistência em Cirurgia Plástica de São Paulo, São Paulo, SP, Brasil. 


\section{INTRODUÇÃO}

A infusão controlada do $\mathrm{CO}_{2}$ é um procedimento não cirúrgico, seguro, que consiste na aplicação do gás carbônico medicinal na pele e no tecido adiposo.

$\mathrm{O}$ aumento da concentração do $\mathrm{CO}_{2}$ diminui o $\mathrm{pH}$, o qual desencadeia uma reação inflamatória, liberando mediadores químicos que estimulam efeitos vasomotores na microcirculação e a síntese de óxido nítrico no local da infusão controlada do $\mathrm{CO}_{2}{ }^{1}$. O aumento da concentração do óxido nítrico induz à neoformação vascular, que juntamente com os fatores de crescimento promovem a fibrogênese na matriz extracelular ${ }^{2}$.

A primeira via de utilização do $\mathrm{CO}_{2}$ foi a percutânea, por meio dos banhos em água enriquecida com gás carbônico termal ou industrial ${ }^{3}$. A via subcutânea foi utilizada secundariamente por Wollina et al. ${ }^{4}$, que observaram diminuição da infecção local em feridas crônicas, assim como a antecipação da cicatrização dessas feridas após aplicação do gás.

Estudos recentes revelaram que a aplicação subcutânea ou transdérmica do $\mathrm{CO}_{2}$ recupera o tecido de granulação em úlceras crônicas, facilitando a integração do enxerto autólogo de pele ${ }^{5}$. Estudos prospectivos têm mostrado diferentes utilizações para o uso do $\mathrm{CO}_{2}$. Assim, o aumento da perfusão tecidual periférica causada pela hipercapnia durante a anestesia reduz a incidência de infecção em feridas cirúrgicas ${ }^{6}$. A segurança da utilização do $\mathrm{CO}_{2}$ está relacionada na literatura pelo seu uso preferencial em relação ao nitrogênio gasoso nas cirurgias videoendoscópicas? ${ }^{7}$ Na atualidade, a utilização do $\mathrm{CO}_{2}$ foi direcionada para procedimentos estéticos, inicialmente no tratamento de gordura localizada e, posteriormente, na melhoria do contorno corporal após lipoaspiração ${ }^{8}$. Da mesma forma, o $\mathrm{CO}_{2}$ também foi empregado no rejuvenescimento facial para tratamento da pálpebra senil e bolsa malar9.

O presente estudo relata os efeitos clínicos e histológicos da infusão controlada do $\mathrm{CO}_{2}$ no tecido de granulação das úlceras crônicas, causadas por insuficiência venosa dos membros inferiores.

\section{MÉTODO}

Foram avaliados 10 pacientes, não fumantes, 7 do sexo feminino e 3 do sexo masculino, com idade média de 56 anos e peso médio de $66,5 \mathrm{~kg}$, portadores de úlceras crônicas causadas por insuficiência venosa dos membros inferiores. O tempo médio de ulceração das feridas foi de 12,5 meses. A Tabela 1 apresenta a distribuição dos pacientes e das áreas ulceradas. As ulcerações não apresentaram sinais clínicos de infecção que necessitassem o uso de antibióticos sistêmicos. Curativos com gaze vaselinada foram trocados diariamente.

Todos os pacientes foram previamente informados e assinaram um termo de consentimento para realização do procedimento, que foi conduzido de acordo com a Declaração de Helsinki.

A aplicação subcutânea do $\mathrm{CO}_{2}$ foi realizada nas ulcerações do membro inferior direito nos 10 pacientes. Três dos 10 pacientes apresentaram ulceração em ambos os membros inferiores, porém a aplicação do $\mathrm{CO}_{2}$ somente foi realizada no membro inferior direito.

A infusão controlada do $\mathrm{CO}_{2}$ foi realizada por um aparelho digital, programável, com unidade de aquecimento do gás. As puncturas foram realizadas a $0,5 \mathrm{~cm}$ da borda da ferida, distando $2,5 \mathrm{~cm}$ entre si, com agulha voltada para o tecido de granulação (Figura 1). O volume do gás infundido foi de $1,5 \mathrm{ml} \mathrm{CO}_{2} / \mathrm{kg}$ de peso ponderal do paciente, por punctura, por sessão. A velocidade do fluxo de infusão do $\mathrm{CO}_{2}$ foi de $80 \mathrm{ml} /$ minuto. As sessões foram repetidas com intervalos de 72 horas, até obter-se a coloração vermelha do tecido de granulação, acompanhada de pequeno sangramento durante a troca do curativo. $\mathrm{O}$ número de sessões foi determinado pela aparência clínica e histológica do tecido de granulação estando apto a receber o enxerto autólogo de pele.

Tabela 1 - Distribuição dos pacientes e características das ulceações.

\begin{tabular}{|c|c|c|c|c|c|c|c|c|c|}
\hline \multirow{3}{*}{\multicolumn{2}{|c|}{ Idade }} & \multirow{3}{*}{$\begin{array}{c}\text { Sexo } \\
\text { feminino }\end{array}$} & \multirow{3}{*}{$\begin{array}{c}\text { Peso (kg) } \\
61\end{array}$} & \multicolumn{6}{|c|}{ Ulceração } \\
\hline & & & & \multicolumn{2}{|c|}{ Ulceração (mês) } & \multicolumn{2}{|c|}{ Tamanho da ferida $(\mathrm{cm})$} & \multicolumn{2}{|c|}{ Largura da ulceração $(\mathrm{cm})$} \\
\hline & & & & $\frac{\text { mmii D }}{12}$ & mmii E & $\begin{array}{c}\text { mmii D } \\
6,5\end{array}$ & $\frac{\text { mmii E }}{-}$ & $\begin{array}{c}\text { mmii } \mathbf{D} \\
3,0\end{array}$ & $\begin{array}{c}\text { mmii E } \\
-\end{array}$ \\
\hline 2 & 59 & feminino & 66 & 14 & - & 7,0 & - & 4,5 & - \\
\hline 3 & 58 & feminino & 64 & 14 & - & 4,5 & - & 3,0 & - \\
\hline 6 & 54 & masculino & 66 & 10 & - & 4,0 & - & 3,0 & - \\
\hline 7 & 56 & masculino & 70 & 15 & 12 & 7,0 & 6,0 & 4,5 & 4,0 \\
\hline 8 & 57 & feminino & 65 & 12 & - & 5,5 & - & 3,0 & - \\
\hline 9 & 55 & feminino & 71 & 10 & - & 6,5 & - & 4,0 & - \\
\hline
\end{tabular}




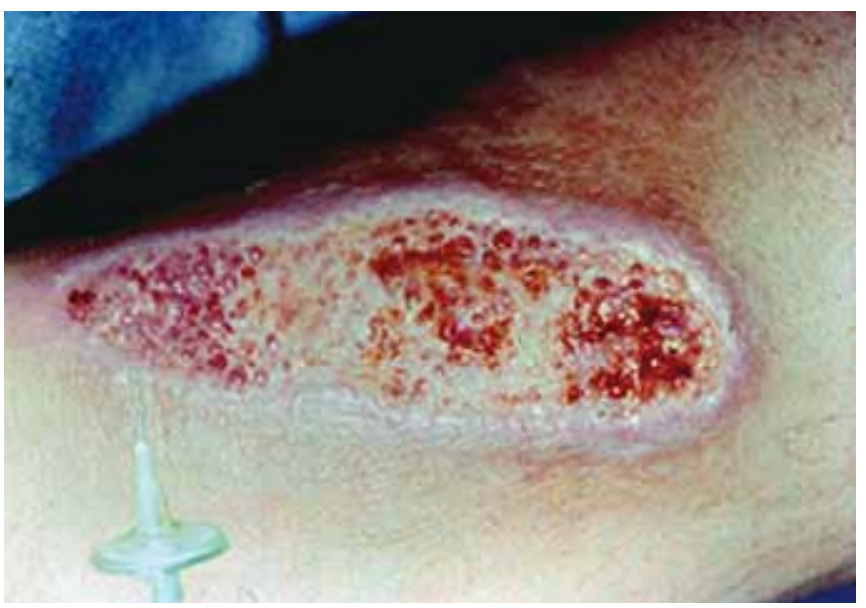

Figura 1 - Carboinsuflação foi realizada com agulha introduzida $0,5 \mathrm{~cm}$ da margem da ferida com inclinação de $45^{\circ} \mathrm{em}$ relação à superficie da pele, direcionada para o centro da mesma.

\section{Análise Histológica}

Biopsias foram realizadas no tecido de granulação de ambos os membros inferiores antes da aplicação controlada do $\mathrm{CO}_{2}$ e 24 horas após a terceira, quinta e décima sessão. Os fragmentos foram fixados em parafina e corados com hematoxilina-eosina. A contagem histológica foi realizada pelo método de Weibel, utilizando uma ocular K10X Zeiss Integrationplatte I, apresentando 25 pontos distribuídos em 5 linhas simétricas com 5 pontos distribuídos geometricamente em cada uma delas. Adilatação das arteríolas pré-capilares foi mensurada usando uma objetiva Zeiss com aumento de 40X. Capilares foram contados usando objetiva Zeiss com aumento de 40X, enquanto macrófagos e fibroblastos com aumento de 100X. Fotos foram feitas com uma câmera digital Nikon DX1200 e digitalizadas em um computador usando o programa Nikon Act-1 Platform versão 2.7.

\section{Análise Estatística}

Os efeitos da infusão controlada do $\mathrm{CO}_{2}$ sobre capilares, macrófagos e fibroblastos foram avaliados pelo teste de Wilcoxon para duas amostras independentes. A análise de variância e múltiplas comparações de Friedman foram aplicadas para avaliar capilares, macrófagos e fibroblastos no tecido de granulação de cada membro inferior e entre eles, antes e após a infusão controlada do $\mathrm{CO}_{2}$ até a décima sessão.

\section{RESULTADOS}

Não foram encontradas diferenças significativas nas dimensões da ferida antes e após a infusão controlada do $\mathrm{CO}_{2} \mathrm{em}$ cada membro inferior. A Tabela 2 demonstra o número de puncturas e volume do $\mathrm{CO}_{2}$ aplicado na ulceração, por sessão, no membro inferior direito. $\mathrm{O}$ volume médio de $\mathrm{CO}_{2}$ aplicado por punctura foi de $99,75 \mathrm{ml}$, com média de 6 puncturas, totalizando um valor médio de $598 \mathrm{ml}$ por ulceração, por sessão.

\begin{tabular}{c|c|c|c|c}
\hline \multicolumn{4}{c}{ Tabela 2 - Puncturas e volume do $\mathrm{CO}_{2}$ aplicadas por ulceração. } \\
\hline \multicolumn{2}{|c|}{} & \multicolumn{3}{|c}{ Infusão do $\mathbf{C O}_{2}\left(\mathbf{1 , 5 m l ~} \mathbf{C O}_{2} / \mathbf{k g}\right)$} \\
\hline & Peso (kg) & Puncturas & mICO$_{2}$ por punctura & mICO$_{2}$ por sessão \\
\hline 1 & 61 & 8 & 91,5 & 732 \\
\hline 2 & 66 & 8 & 99 & 792 \\
\hline 3 & 64 & 4 & 96 & 384 \\
\hline 4 & 71 & 6 & 106,5 & 639 \\
\hline 5 & 63 & 4 & 94,5 & 378 \\
\hline 6 & 65 & 4 & 97,5 & 370 \\
\hline 7 & 70 & 8 & 105 & 840 \\
\hline 8 & 65 & 6 & 97,5 & 585 \\
\hline 9 & 71 & 6 & 106,5 & 639 \\
\hline 10 & 69 & 6 & 103,5 & 621 \\
\hline
\end{tabular}

Todas as feridas no membro inferior direito apresentaram melhoria do tecido de granulação, caracterizada por diminuição do edema, mudança na coloração de rosa pálido a vermelha intensa, com pequenos pontos de sangramento nas trocas de curativo (Figura 2). O tecido de granulação das ulcerações no membro inferior direito foi considerado apto a receber enxerto autólogo de pele após dez sessões da infusão controlada do $\mathrm{CO}_{2}$ (Figura 3). Nenhuma ferida no membro inferior esquerdo tornou-se apta a receber enxerto no mesmo período de tempo.
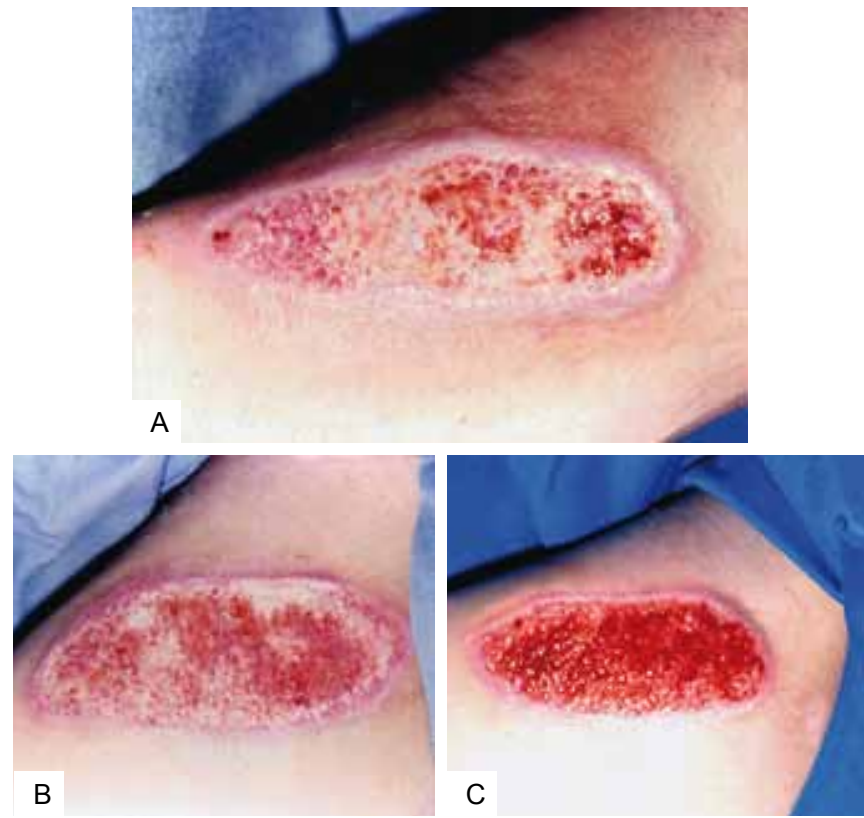

Figura 2 - A: Úlcera crônica do membro inferior direito com intenso depósito de fibrina e edema no leito do tecido de granulação. B: Diminuição do edema com recuperação do tecido de granulação após a quinta sessão da infusão controlada do $C O$. C: Tecido de granulação apto a receber o enxerto autólogo de pele após dez sessões. 

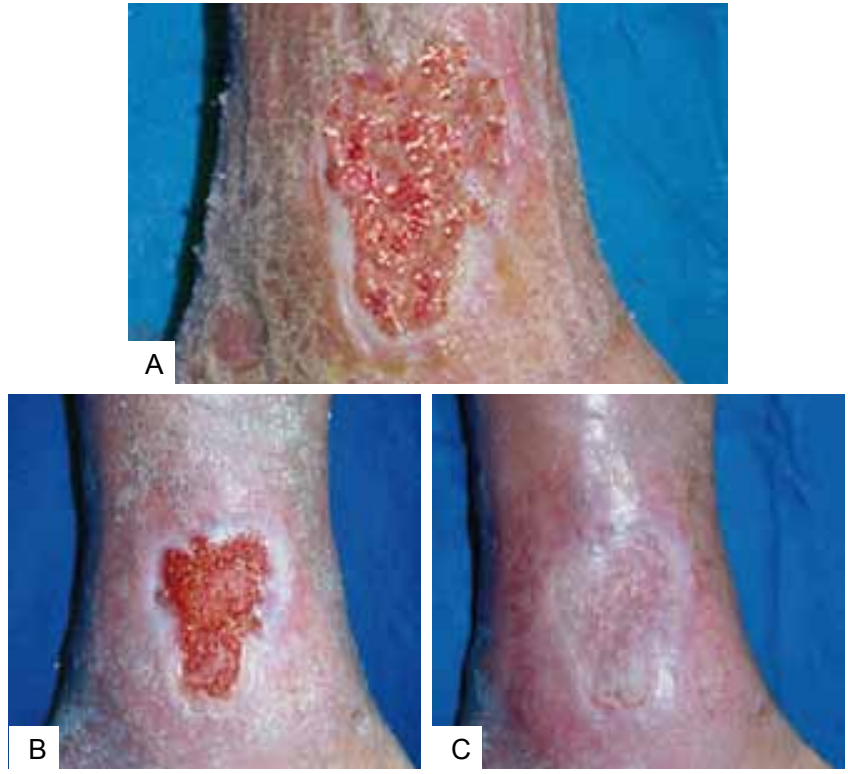

Figura 3 - A: Úlcera na face interna da perna junto ao maléolo medial, edemaciada, bordas irregulares, com feixes de fibrina no leito da ulceração. Intensa serosidade sobre a pele circunvizinha. B: Aspecto da ulceração após 10 sessões da infusão controlada do $\mathrm{CO}_{2}$, demonstrando o tecido de granulação apto a receber enxerto autólogo de pele. $\boldsymbol{C}$ : Integração do enxerto autólogo de pele após 15 dias da sua aplicação.

A Tabela 3 exibe os valores da dilatação das art ríolas pré-capilares antes e após a infusão controlada do $\mathrm{CO}_{2}$, em ambos os membros inferiores. $\mathrm{O}$ diâmetro médio dos vasos no membro inferior direito foi 3,4 vezes maior após a infusão controlada do $\mathrm{CO}_{2}$ (Figura 4). Ao mesmo tempo, houve aumento de 3,1 vezes no diâmetro das arteríolas pré-capilares do membro inferior direito em relação às do membro inferior esquerdo, no mesmo período de avaliação.

A Tabela 4 apresenta o comportamento dos capilares, macrófagos e fibroblastos na granulação tecidual em ambos os membros inferiores. O número de capilares foi significantemente maior $(p<0,01)$ no membro inferior direito após infusão de $\mathrm{CO}_{2}$ e também, significantemente maior $(p<0,01)$ no membro inferior direito em relação ao membro inferior esquerdo (Figura 5). O aumento dos capilares ocorreu entre a terceira e décima sessão. $\mathrm{O}$ número de macrófagos e fibroblastos foi significante maior $(\mathrm{p}<0,05)$ no membro inferior direito após a infusão controlada do $\mathrm{CO}_{2}$. Eles mostraram aumento significante $(\mathrm{p}<0,05)$ no membro inferior direito em relação ao esquerdo (Figuras 6 e 7). O número de macrófagos aumentou a partir da terceira sessão, diminuindo a partir da quinta sessão, enquanto os fibroblastos apresentaram grande aumento após a quinta sessão. Não foi observada mudança significativa no número de capilares, macrófagos e fibroblastos no membro inferior esquerdo no mesmo período de avaliação.

\begin{tabular}{|c|c|c|c|c|}
\hline & \multicolumn{2}{|c|}{ Antes da infusão de $\mathrm{CO}_{2}$} & \multicolumn{2}{|c|}{24 h após $3^{\mathrm{a}}$ infusão de $\mathrm{CO}_{2}$} \\
\hline & mmii D & mmii E & mmii D & mmii E \\
\hline 1 & 11,8 & 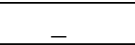 & 41,2 & \\
\hline 2 & 29,1 & - & 96,4 & - \\
\hline 3 & 42 & - & 124,3 & \\
\hline 4 & 35,3 & 31,2 & 94,2 & 29,1 \\
\hline 5 & 29,1 & - & 141,2 & - \\
\hline 6 & 11,8 & & 47,6 & \\
\hline 7 & 35,3 & 34,6 & 105,8 & 33,1 \\
\hline 8 & 31,6 & $\ldots$ & 125,2 & - \\
\hline 9 & 15,5 & - & 38,5 & \\
\hline 10 & 25,8 & 26,5 & 95,4 & 26,2 \\
\hline
\end{tabular}
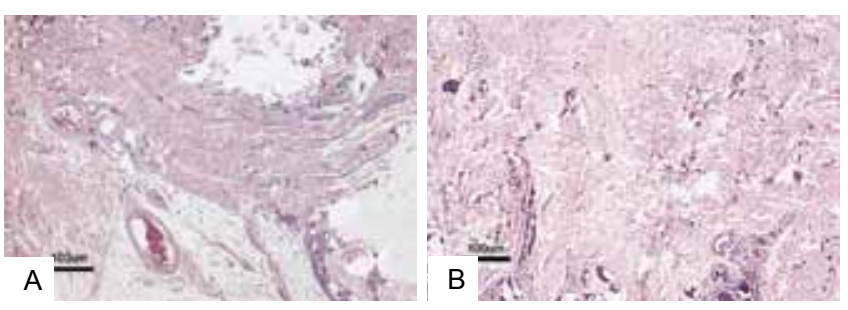

Figura 4 - A: Aspecto do diâmetro do vaso antes da infusão do $\mathrm{CO}_{2}$. Dilatação dos capilares após a infusão controlada do $\mathrm{CO}_{2}$.

\begin{tabular}{c|c|c|c|c|c|c}
\hline \multicolumn{6}{c}{ Tabela 4 - Frequência da média de capilares, macrófagos e } \\
fibroblastos no tecido de granulação. \\
\hline & \multicolumn{2}{c}{ Capilares } & \multicolumn{1}{c}{ Macrófagos } & \multicolumn{2}{c}{ Fibroblastos } \\
\cline { 2 - 7 } & mmii D & mmii E & mmii D & mmii E & mmii D & mmii E \\
\hline antes aplicação $\mathrm{CO}_{2}$ & 15 & 12 & 57 & 50 & 19 & 25 \\
\hline após 3 ${ }^{\text {a }}$ aplicação & 29 & 18 & 99 & 50 & 41 & 21 \\
\hline após 5 $5^{\text {a } \text { aplicação }}$ & 51 & 17 & 46 & 58 & 88 & 20 \\
\hline após $10^{\text {a }}$ aplicação & 107 & 11 & 48 & 51 & 63 & 27 \\
\hline
\end{tabular}
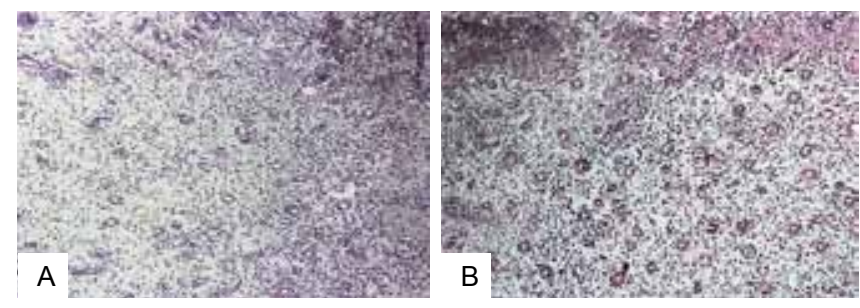

Figura 5-A: Distribuição dos capilares no membro inferior esquerdo onde não foi aplicado o $\mathrm{CO}_{2}$. B: Expressivo aumento do número de capilares após cinco sessões, correspondente ao mesmo periodo da histologia da Figura 5 A. 


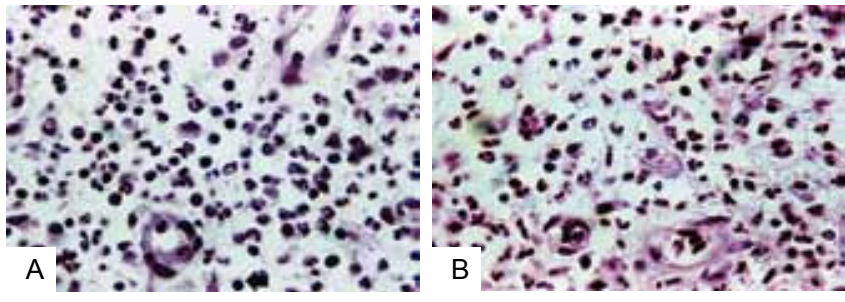

Figura 6 - A: Tecido de granulação antes da infusão controlada do $\mathrm{CO}_{2}$ exibindo grande quantidade de leucócitos polimorfonucleares em relação aos macrófagos. A seta aponta um macrófago típico. B: Aumento significante do número de macrófagos após a terceira sessão.
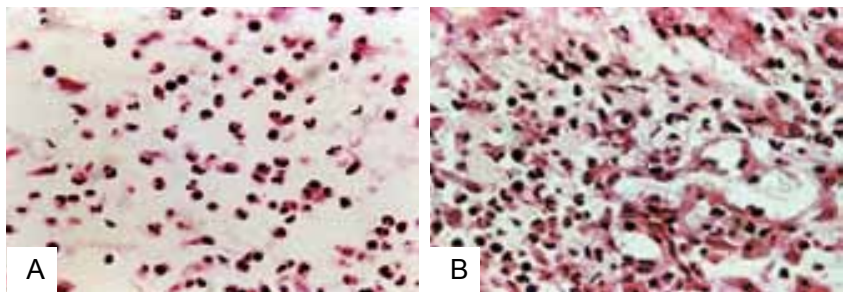

Figura 7 - A: Edema do tecido de granulação promovendo dispersão das células inflamatórias com predominância de polimorfonucleares e macrófagos. B: Aumento dos

fibroblastos na matriz extracelular no tecido de granulação após a quinta sessão.

\section{DISCUSSÃO}

$\mathrm{O}$ aumento da concentração do $\mathrm{CO}_{2}$, assim como a elevação do hidrogênio iônico, liberado na reação do $\mathrm{CO}_{2}$ com a $\mathrm{H}_{2} \mathrm{O}$, no local da infusão, são responsáveis pela diminuição do $\mathrm{pH}$, desencadeando respostas inflamatórias de natureza diversa nos tecidos infundidos. A diminuição do $\mathrm{pH}$ potencializou o efeito Bohr no interior da hemácia, desviando para a direita a curva de dissociação do oxigênio da hemoglobina e, com isso, aumentou a oferta de oxigênio ao tecido de granulação das úlceras crônicas nos membros inferiores ${ }^{10}$. A resposta vascular se caracterizou por um efeito vasomotor e pela formação de novos vasos sanguíneos, aumentando o fluxo sanguíneo no local da aplicação do gás. A diminuição do pH também estimulou os receptores de membrana do sistema nervoso autônomo parassimpático a liberar seu neurotransmissor, a acetilcolina ${ }^{11}$. A acetilcolina promoveu a hidrólise da miosina do músculo liso do vaso, diminuindo a resistência da parede vascular e, com isso, dilatou as arteríolas précapilares do tecido de granulação nas úlceras crônicas dos membros inferiores ${ }^{11}$.

No presente estudo, a perda de resistência da parede do vaso aumentou 3,4 vezes o diâmetro das arteríolas pré-capilares no tecido de granulação. De acordo com
Papapetropoulos et al. ${ }^{2}$, a neoformação vascular estimulada pelo óxido nítrico, mediadores químicos e fatores de crescimento, liberados nos tecidos após a aplicação do $\mathrm{CO}_{2}$, foi evidenciada pelo aumento da densidade capilar no tecido de granulação. Toriyama et al. ${ }^{12}$ também descreveram a atuação dos fatores de crescimento, em especial o fator vascular de crescimento endotelial, na formação de novos vasos sanguíneos, após banhos em água carbonada.

A vasodilatação das arteríolas pré-capilares, associada à neoformação vascular e à potencialização do efeito Bohr, aumentou o fluxo sanguíneo, a oferta de oxigênio aos tecidos e reduziram o edema no tecido de granulação da ferida cutânea crônica, tornando-a apta a receber o enxerto autólogo de pele. O fechamento espontâneo das úlceras crônicas dos membros inferiores não ocorreu no presente estudo, em razão da persistência da insuficiência venosa nos mesmos.

O aumento do fluxo sanguíneo na microcirculação após exposição da pele ao $\mathrm{CO}_{2}$ foi observado por Schnizer et al. ${ }^{13}$, por meio da fluxometria com laser Doppler. A resposta celular foi desencadeada pelo aumento da porosidade da parede vascular em razão da sua dilatação. A migração de células inflamatórias, em especial macrófagos, fibroblastos e células endoteliais progenitoras, ocorreu através dos poros dilatados das arteríolas pré-capilares ${ }^{14}$. A quimiotaxia das células inflamatórias para o local de aplicação do $\mathrm{CO}_{2}$, orientada pelo fator de crescimento do fibroblasto básico, fator vascular de crescimento endotelial e fator de crescimento transformador alfa e beta, promoveu a fibrogênese na matriz extracelular do tecido de granulação. D'Arcangelo et al. ${ }^{15}$ descreveram a influência da acidose induzida pela hipercapnia e a distensão tecidual promovida pelo $\mathrm{CO}_{2}$ na atividade dos fatores de crescimento do fibroblasto básico e transformador beta na fibrogênese. A diminuição no número de macrófagos, acompanhada pelo aumento de fibroblastos, após a quinta aplicação controlada do $\mathrm{CO}_{2}$, comprova a diferenciação celular estimulada pelo fator de crescimento do fibroblasto básico e fator de crescimento transformador beta. $\mathrm{O}$ fator de crescimento transformador beta promoveu o desenvolvimento de fibroblastos em fibras colágenas e elásticas, fornecendo maior sustentação para o tecido de granulação. A diminuição dos fibroblastos após a quinta aplicação da infusão controlada do $\mathrm{CO}_{2}$, encontrada no presente estudo, está relacionada com o aumento das fibras na matriz extracelular do tecido de granulação.

\section{CONCLUSÃO}

Concluindo, a somatória das respostas vasomotora e celular, estimuladas pela infusão controlada de $\mathrm{CO}_{2}$, permitiu o fechamento espontâneo das úlceras de pequena extensão ou tornou-as aptas a receber o enxerto autólogo de pele, nas de média e grande extensão. 


\section{REFERÊNCIAS}

1. Brockow T, Hausner T, Dillner A, Resch KL. Clinical evidence of subcutaneous $\mathrm{CO}_{2}$ insufflations: a systematic review. J Altern Complement Med. 2000;6(5):391-403.

2. Papapetropoulos A, García-Cardenã G, Madri JA, Sessa WC. Nitric oxide production contributes to the angiogenic properties of vascular endothelial growth factor in human endothelial cells. J Clin Invest. 1997;100(12):3131-9.

3. Schmidt J, Monnet P, Normand B, Fabry R. Microcirculatory and clinical effects of serial percutaneous application of carbon dioxide in primary and secondary Raynauld's phenomenon. Vasa. 2005;34(2):93100.

4. Wollina U, Heinig B, Uhlemann C. Transdermal $\mathrm{CO}_{2}$ application in chronic wounds. Int J Low Extrem Wounds. 2004;3(2):103-6.

5. Abramo AC, Ledo-Silva M. Carbon dioxide infusion: clinical and histological appraisal in chronic wounds. Australian New Zeland Clinical Trials. ACTRN 12609001038280. 2009.

6. Akça O, Doufas AG, Morioka N, Iscoe S, Fisher J, Sessier DI. Hypercapnia improves tissue oxygenation. Anesthesiology. 2002;97(4):8016.

7. Hanly EJ, Fuentes JM, Aurora AR, Bachman SL, De Maio A, Marohn MR, et al. Carbon dioxide pneumoperitoneum prevents mortality from sepsis. Surg Endosc. 2006;20(9):1482-7.

8. Brandi C, D'Aniello C, Grimaldi L, Caiazzo E, Stanghellini E. Carbon dioxide therapy: effects on skin irregularity and its use as a complement to liposuction. Aesthetic Plast Surg. 2004;28(4):222-5.
9. Abramo AC. Periorbital rejuvenation by $\mathrm{CO}_{2}$ infusion. In: Rejuvenation of the eyes: medical and surgical approaches. Session 13. Congress Book. Anti-Aging Medicine World Congress. Global Aging Management. Preventive and Longevity Medicine, Aesthetic Dermatology and Surgery. $6^{\text {th }}$ ed. Paris; 2008. p.66-7.

10. Jensen FB. Red blood cell $\mathrm{pH}$, the Bohr effect, and other oxygenationlinked phenomena in blood $\mathrm{O}_{2}$ and $\mathrm{CO}_{2}$ transport. Acta Physiol Scand. 2004;182(3):215-27.

11. Abramo AC. Sistema nervoso autônomo e a hemodinâmica do meio interno após a infusão do $\mathrm{CO}_{2}$. In: Abramo $\mathrm{AC}$, ed. Infusão controlada do $\mathrm{CO}_{2}$ : carboinsuflação. $1^{\mathrm{a}}$ ed. São Paulo:2010. p.54-63.

12. Toriyama T, Kumada Y, Matsubara T, Murata A, Ogino A, Hayashi H, et al. Effect of artificial carbon dioxide foot bathing on critical limb ischemia (Fontaine IV) in peripheral arterial disease patients. Int Angiol. 2002;21(4):367-73.

13. Schnizer W, Erdl R, Schöps P, Seichert N. The effect of external $\mathrm{CO}_{2}$ application on human skin microcirculation investigated by laser Doppler flowmetry. Int J Microcirc Clin Exp. 1985;4(4):343-50.

14. Abramo AC. Resposta inflamatória celular à infusão tecidual do $\mathrm{CO}_{2}$. In: Abramo AC, ed. Infusão controlada do $\mathrm{CO}_{2}$ : carboinsuflação. $1^{\mathrm{a}}$ ed. São Paulo:2010. p.115-29.

15. D'Arcangelo D, Facchiano F, Barlucci LM, Melillo G, Illi B, Testolin $\mathrm{L}$, et al. Acidosis inhibits endothelial cell apoptosis and function and induces basic fibroblast growth factor and vascular endothelial growth factor expression. Circ Res. 2000;86(3):312-8.

\section{Correspondência para:}

Antonio Carlos Abramo

ACA - Instituto de Assistência em Cirurgia Plástica de São Paulo

Rua Afonso de Freitas, 641 - Paraíso - São Paulo, SP, Brasil - CEP 04006-052

E-mail: acabramo@abramo.com.br 